\title{
Le bassin versant expérimental du Torrent de l'Eglise (Les Arcs - Savoie)
}

\author{
Structure, flux, dynamique
}

\author{
H. Vivian \\ Responsable scientifique (Laboratoire de la Montagne Alpine - URA 344 Grenoble)
}

\author{
G. Bocquet*, J.-P. Dedieu*, D. Fabre**, A. Thomas*, J.-Cl. Thouret*
}

Membres de l'équipe de recherche

\begin{abstract}
Une des actions de recherches soutenue dans le domaine des risques torrentiels par le Contrat Etat-Région RhôneAlpes de 1987 à 1990, a été l'installation et la surveillance d'un bassin versant expérimental où les phénomènes d'érosion torrentielle et de mouvements de versant, tributaires de toute la dynamique du bassin, étaient susceptibles d'être présents et actifs.

Pour cela, le choix s'est porté sur le bassin du Torrent de l'Eglise situé sur le versant des Arcs (Bourg-St-Maurice) voisin immédiat de celui de la Ravoire qui, en 1981, a connu une crue et une lave torrentielle exceptionnelles.
\end{abstract}

* URA 344, 17, rue M. Gignoux, 38031 Grenoble Cedex.

** IRIGM, Domaine Universitaire, Saint-Martin-d'Hères.

\section{I - CONTEXTE GÉOGRAPHIQUE GÉNÉRAL DU SITE ET ÉQUIPEMENTS IMPLANTÉS POUR L'ÉTUDE}

\begin{abstract}
A la limite de la zone périphérique du Parc National de la Vanoise, le bassin de Bourg-St-Maurice fait partie de la zone de transition en Tarentaise entre les domaines alpins intermédiaires et alpins internes. Le changement de direction de la vallée de l'Isère vers le sud-ouest entre SainteFoy et Bourg-St-Maurice, engendre un nouvel éventail d'expositions sur les deux rives, tournées l'une vers le Sud-Est et l'autre vers le Nord-Ouest.

En rive gauche, le versant de la "Montagne des Arcs " exposé au Nord-Ouest est parcouru par un réseau de
\end{abstract}

\section{The experimental catchment area of the torrent of l'Eglise (Les Arcs - Savoie) Structure, flows, dynamics}

One of the research actions taken in the field of torrential risks as part of the contract between the State and the Rhones-Alpes Region was the installation and monitoring of an experimental catchment area in which phenomena of torrential erosion and catchment area movements, dependent on the entire dynamics of the catchment area, were likely to be present. For this the catchment area of the torrent of l'Eglise on the slopes of the Arcs, Bourg-St-Maurice was chosen. This is right beside the Ravoire torrent which experienced exceptional high water and torrential lava levels in 1981. The article presents the geographic context of the site, the equipment installed for the study, the research team and the scientific problem, and finally results of the study. 


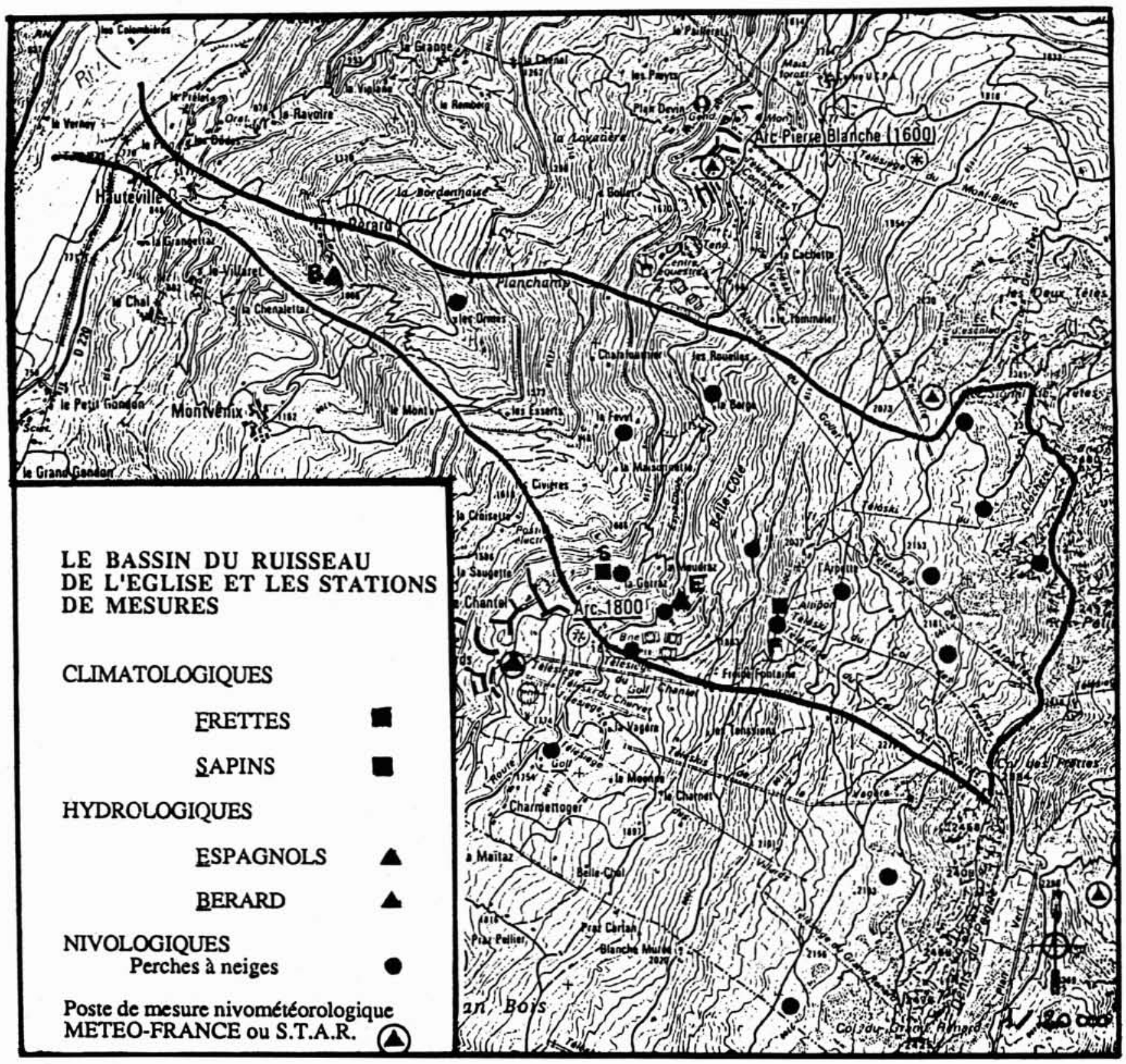

torrents subparallèles (La Ravoire, l'Eglise, le StPantaléon, le Villard). Le bassin du Torrent de l'Eglise a été choisi pour nos recherches. En plan, le bassin de 800 à $2500 \mathrm{~m}$ d'altitude et recouvrant approximativement $5 \mathrm{~km}^{2}$ revêt une forme de cône dont l'impluvium supérieur est largement ouvert en amphithéâtre sur une largeur de $2 \mathrm{~km}$ et dont la partie terminale à l'aval n'a plus que $500 \mathrm{~m}$ de large. Il est creusé dans les schistes du Houiller (zone subbriançonnaise) plus ou moins altérés. Seule la partie supérieure incluant la crête culminant à l'Aiguille Grive $(2735 \mathrm{~m})$ est constituée de quartzites et de gneiss (zone briançonnaise).

En position d'ubac, ce bassin fortement incliné (30\% en moyenne) connaît un climat rude marqué par des températures annuelles moyennes s'étageant de $7^{\circ} \mathrm{C}$ à moins de $4^{\circ} \mathrm{C}$ et où une année moyenne comporte de 4 à 5 mois froids $\left(<7^{\circ} \mathrm{C}\right)$ dans le bas du versant, à $8-9$ mois dans le haut (carte climatique détaillée au $1 / 250000^{\mathrm{e}}$ - E.R. $30-$ Feuille Annecy - Thonon (1978-79). La pluviométrie très moyenne $(1000 \mathrm{~mm})$, sans grande inégalité saisonnière, comporte une importante part de neige dont la fusion apporte l'abondance aux écoulements printaniers. Les ruisseaux ou torrents qui dévalent du haut du versant ne sont pas très impétueux, la plupart du temps, mais capables de paroxysmes, ainsi que la crue de 1981 et la lave torrentielle de la Ravoire l'ont démontré. Toutefois, il est remarquable de noter que dans les archives du Service RTM de Savoie et que dans l'ouvrage sur les torrents de Savoie de P. Mougin (1914), les écoulements du versant des Arcs ne sont pas notés comme dangereux et sont même absents du palmarès des crues torrentielles calamiteuses. La formation de la lave exceptionnelle de la Ravoire en 1981 n'est pas encore entièrement expliquée quant aux facteurs qui l'auraient déclenchée. Essayer donc de détecter sur le bassin voisin des signes marqués d'érosion, de mettre en interrelation les divers paramètres susceptibles de créer une situation de fragilité accrue, mesurer et enfin effectuer une première approche de l'hydrologie du torrent n'en présentait que plus d'intérêts (aucune mesure de débit n'était régulièrement effectuée sur ce versant jusque-là).

La végétation s'étage selon un schéma classique lié au versant d'ubac avec, sur le cône de déjection, des cultures 
jouxtant le village de Hauteville-Gondon, puis des formations forestières parfois assez denses jusque vers $1500 \mathrm{~m}$, et enfin, sur l'escarpement majeur du bassin, une zone à prédominance de landes précédant celle des alpages, sur le gradin supérieur au pied des crêtes. Cette dernière zone supporte l'essentiel du domaine skiable des stations d'Arc 1600 et Arc 1800.

Sur ce bassin, dès 1987, la première action a été l'implantation de stations de mesure pour quantifier des paramètres climatologiques et hydrologiques dont la connaissance est, avec celles des paramètres de structure, indispensables pour l'étude.

Ont donc été installées 2 stations climatologiques, dites des Sapins (1 $735 \mathrm{~m})$ et des Frettes (2035 m) complétant en altitude les mesures effectuées à Bourg-St-Maurice par la station de la Météorologie Nationale. Etant donnée la grande part que prend la neige dans les précipitations et l'omniprésence d'un manteau nival pendant plusieurs mois, un réseau de 14 perches à neige a été implanté dans le bassin supérieur essentiellement, la perche la plus basse étant vers $1300 \mathrm{~m}$, la plus haute à $2300 \mathrm{~m}$. Le but de ces mesures de hauteur et de densité effectuées régulièrement au cours de 3 hivers était de préciser la part du stock nival dans la formation des forts débits au moment de la fusion ainsi que son rôle de rétention pendant la saison hivernale.

Les débits ont été mesurés à 3 stations hydrométriques :

- une station dans le bassin supérieur à $1790 \mathrm{~m}$, «les Espagnols-amont " du nom du chemin qui traverse le bassin à cet endroit ;

- une station, toujours dans le site des Espagnols, "Espagnols-aval » devait mesurer le débit résiduel liant bassin supérieur $\left(3 \mathrm{~km}^{2}\right)$ et bassin inférieur après un captage de la conduite EDF installée au lendemain de la crue de la Ravoire et destinée à écrêter les crues de fréquence quinquennale des torrents de tout le versant des Arcs ;

- une troisième station dans le bassin inférieur près du hameau du Bérard à $1060 \mathrm{~m}$ d'altitude.

\section{II - CONSTITUTION DE L'ÉQUIPE DE RECHERCHE ET PROBLÉMATIQUE SCIENTIFIQUE}

L'équipe engagée dans cette action scientifique, par ailleurs soutenue par l'Université Joseph Fourier (Grenoble) et la commune de Bourg-St-Maurice, sous la responsabilité d'Huguette Vivian (Directeur de Recherche à l'URA 344) a été essentiellement constituée par les géographes physiciens du Laboratoire de la Montagne Alpine, un chercheur de l'Institut de Recherche Interdisciplinaire de Géologie et de Mécanique et, à l'occasion des diverses recherches entreprises, des collaborateurs du Laboratoire de Biologie Végétale, de l'Institut Dolomieu (tous, laboratoires grenoblois) et de l'Université de Sapporo (Japon). Au total l'équipe a compris 6 chercheurs permanents auxquels sont venus s'adjoindre une douzaine de participants temporaires.

La variété des recherches et des spécificités de tous les membres de l'équipe ont contribué à la réalisation d'une étude globale du bassin-versant. La géologie, la lithologie, l'hydrogéologie, la morphologie, la climatologie, la nivologie, l'hydrologie, la végétation et l'occupation humaine ont été étudiées et cartographiées au $1 / 5000^{\mathrm{C}}$. De cet ensemble de recherches thématiques effectuées dans le même esprit, il a été possible d'opérer une synthèse et d'en déduire la dynamique des zones sensibles actuelles et potentielles. Il s'agit donc d'une prise en compte, la plus complète possible, des paramètres du milieu pour étudier leurs interrelations ainsi que le fonctionnement du bassin dans le double but :

- d'une recherche fondamentale sur la stabilité des versants et l'érosion torrentielle ;

- d'une recherche appliquée: la rédaction de documents cartographiques analytiques et synthétiques obtenus selon la méthode classique de cartographie ou par cartographie automatique aboutissant à une carte de synthèse des risques pouvant être considérée comme un outil d'aide à la décision.

\section{III - LES RÉSULTATS}

Nombreux ont été les problèmes et trop court le temps attribué à cette vaste recherche pour laquelle il manquera un suivi permettant de corroborer certaines hypothèses, une actualisation incessante des diverses données permettant de comprendre la dynamique du bassin dans toutes ses dimensions spatio-temporelles, des recherches plus approfondies concernant certains points de la géophysique, de l'hydrogéologie, de l'étude de l'humidité dans le sol, du rôle de la fusion nivale dans l'alimentation en eau du sol et celle des crues torrentielles, etc... Pour ces dernières recherches qui nous paraissent essentielles, il faut préciser que nous avons "vécu» notre étude dans un contexte climatologique d'années déficitaires où aucun paroxysme ne pouvait être généré !

L'étude globale et intégrée du bassin-versant est exposée dans un rapport rédigé à l'automne 1990 auquel est joint un Atlas regroupant 11 documents cartographiques, 8 noir et blanc, 3 couleurs, reproduits au $1 / 10000^{\mathrm{c}}$ mais rédigés originellement au $1 / 5000^{\mathrm{e}}$.

Le plan du rapport, correspondant au déroulement logique de la pensée et des recherches, se compose de 3 parties.

A - Composantes, éléments et paramètres du bassin versant.

I - Les paramètres de structures.

- caractéristiques topographiques et lithologiques ;

— unité de relief, substrat, formations superficielles et sol ;

- caractéristiques géotechniques ;

- les principales formations végétales ;

- occupation et utilisation du sol.

II - Les paramètres de flux.

- les paramètres climatologiques et nivologiques (installation de 2 stations climatologiques et d'un réseau de perches à neige) ;

- les écoulements superficiels et souterrains (installation de 3 stations hydrométriques). 
B - L'évolution morphodynamique du bassin-versant.

I - Les formes dynamiques ou d'érosions actuelles et récentes.

II - Le chenal du torrent : érosion et évolution.

III - Zone et étagement des processus et de l'instabilité morphodynamique.

C - Diagnostic des instabilités morphodynamique, hydrologique, biogéographique et anthropique.

I - Cartographie assistée par ordinateur vers une représentation synthétique des zones sensibles.

II - Diagnostic des instabilités : outils et critères.

III - Méthode et résultats de la cartographie géoécologique intégrée.

- paramètres ;

- taxo-chorologie des unités spatiales isoschèmes ;

- classification et zonage des types d'instabilité.

Ce rapport (163 p.) est illustré par 56 figures, 17 planches photographiques et 53 tableaux.

Les principales conclusions de ce travail peuvent être résumées ici.

\section{IV - CONSTATS ET PROSPECTIVES}

\section{1 - A propos des paramètres de flux}

Avec un réseau hydrographique perturbé par les actions anthropiques, une grande complexité des entrées et des sorties de l'eau dans le bassin-versant tant en surface qu'au niveau de la circulation souterraine, à laquelle s'ajoutent les phénomènes de rétention dans le sol et de rétention nivale, comprendre le fonctionnement hydrologique $d u$ système "Torrent de l'Eglise " aurait nécessité plus d'équipements de mesures et plus d'années d'observations. Notons que tous les bassins-versants expérimentaux français connus ont entre 10 et 25 ans de fonctionnement (Orgeval, Draix, bassins vosgiens...).

De plus, le hasard a voulu que pour nos années de travail, nous n'avons connu majoritairement que des périodes déficitaires à l'issue desquelles, précisément, l'eau a eu tendance à manquer dans le bassin-versant...

Afin de pouvoir jauger une crue d'importance, en aval, au Bérard, nous avions dû faire concevoir un double seuil de jaugeage capable de laisser passer un débit de $4 \mathrm{~m}^{3} / \mathrm{s}$, comme un très petit débit de type hivernal (le chiffre de $4 \mathrm{~m}^{3} / \mathrm{s}$ avait été avancé à propos de la crue de la Ravoire). Le débit maximum que nous avons pu enregistrer à cette station du Bérard est de $0,24 \mathrm{~m}^{3} / \mathrm{s}$ moyenne journalière le 14 février 1990 avec une pointe de $0,30 \mathrm{~m}^{3} / \mathrm{s}$ le 15 février, un débit moyen journalier de $0,28 \mathrm{~m}^{3} / \mathrm{s}$ le 16 mai 1988 , avec une pointe de $0,31 \mathrm{~m}^{3} / \mathrm{s}$, et enfin encore $0,28 \mathrm{~m}^{3} / \mathrm{s}$ le 19 juin 1988 avec une pointe de $0,30 \mathrm{~m}^{3} / \mathrm{s}$. Ces débits faibles ont été engendrés suite à des séquences de précipitations variant de 40 à $60 \mathrm{~mm}$ en 3-4 jours en mai et juin, et de $218 \mathrm{~mm}$ en 3 jours, dont $84 \mathrm{~mm}$ en $24 \mathrm{~h}$, à la mi-février 1990 , soit en période de rétention nivale. Il est certain que si ce dernier total était tombé sous forme de pluie, en saison estivale, il aurait été possible de mesurer un fort débit. Malheureusement il n'y a pas eu d'épisodes estivaux permettant d'étudier (sans avoir à prendre en compte le rôle complexe de la couverture nivale), le temps de propagation de l'eau dans le bassin supérieur, le temps de réponse du torrent entre les Espagnols et le Bérard, etc...

En fait, même dans des conditions optimales pour la compréhension d'un transfert pluie-débit, nous ne sommes pas sûrs de pouvoir arriver à une hypothèse valable, tant les aménagements faussent le drainage naturel.

Lors de ces petites crues précédemment notées, il est remarquable, ainsi que nous l'avions supposé en décrivant l'hydrographie artificielle, que le rapport entre le bassin supérieur et le bassin inférieur est faible ou même absent. Normalement, la pointe de crue aux Espagnols devrait précéder celle du Bérard. C'est le cas en juin 1990 où effectivement le débit journalier maximum de $0,18 \mathrm{~m}^{3} / \mathrm{s}$ aux Espagnols arrive le 11 juin et de $0,24 \mathrm{~m}^{3} / \mathrm{s}$ au Bérard le 14 ; soit un temps qui paraît long pour la distance... En mai 1988 le débit de $0,13 \mathrm{~m}^{3} / \mathrm{s}$ ne passe aux Espagnols que le 18 , alors qu'au Bérard $0,28 \mathrm{~m}^{3} / \mathrm{s}$ ont été enregistrés le 16 ! De façon identique, en juin $19880,28 \mathrm{~m}^{3} / \mathrm{s}$ sont mesurés au Bérard le 19 juin et c'est seulement le 25 que $0,14 \mathrm{~m}^{2} / \mathrm{s}$ arrive aux Espagnols amont. Comment tenter d'expliquer de tels phénomènes? Il faut évoquer de multiples causes parmi lesquelles :

- le rôle tampon du bassin supérieur, par sa nature même, rôle signalé maintes fois (chapitres lithologie, morphologie, hydrologie);

- le rôle concentrateur des drainages et des canalisations de routes qui déversent les eaux de ruissellement dans le bassin inférieur. Celui-ci reçoit également avant le Bérard les arrivées des eaux pluviales d'Arc 1800 et d'Arc 1600. Non seulement la crue est alimentée par des apports extérieurs au bassin mais elle est artificiellement accélérée!

Il faut noter qu'il est possible d'avoir des totaux pluviométriques journaliers exceptionnels de 80 à $90 \mathrm{~mm}$ (déjà enregistrés en 1981 et 1990 mais toujours en période hivernale), totaux susceptibles de déclencher des crues dans le bassin supérieur, à condition que les autres paramètres favorables à un écoulement massif, essentiellement l'épaisseur et l'état de la couverture neigeuse, soient présents. Cela n'a pas été le cas en 1990.

Enfin, la présence de la dérivation E.D.F. semble amoindrir définitivement les possibilités de grosses crues dans le bassin inférieur. Cette dérivation capable d'encaisser une valeur de $1,2 \mathrm{~m}^{3} / \mathrm{s}$ aux Espagnols, $0,9 \mathrm{~m}^{3} / \mathrm{s}$ au Fevet (soit quelques $2 \mathrm{~m}^{3} / \mathrm{s}$ ) débits qui sont donc détournés du torrent inférieur mesuré au Bérard, fonctionne en permanence; de ce fait, seule une crue de fréquence supérieure à la valeur quinquennale, serait susceptible de relier les deux bassins en passant au-dessus de la route; du moins peut-on le supposer car les transports solides mobilisés à cette occasion pourraient combler la dérivation avant que le débit maximum calculé par S.O.G.R.E.A.H. soit atteint. Alors peut-être serait-il possible de voir le débordement s'effectuer à moins de $1,2 \mathrm{~m}^{3} / \mathrm{s}$ ? 
Compte tenu de toutes les observations que nous avons pu faire, il n'est pas impossible, pour étayer l'étude ultérieure des risques, de concevoir quelques scénarios. En fait, il apparaît que l'eau et son action érosive se manifestent plus sous forme d'écoulements diffus et hypodermiques que sous forme de ruissellement concentré. D'où la possibilité qui nous a été donnée de constater des formes d'érosion de versants alors que le débit du torrent était insignifiant.

Cette dynamique de versant due à la permanence de l'eau ou à la saturation rapide du sol, nous aurions pu la préciser en effectuant une étude de l'évolution spatiotemporelle de l'humidité.

Nous avons l'intention, dans le futur, d'expérimenter deux méthodes indirectes de la détermination de la présence de l'eau et de son rôle dans les mouvements de terrain et les manifestations d'érosion; ce sont :

- d'une part, les études par photographies aériennes infrarouge ou par clichés SPOT que nous avons expérimentées mais qu'il aurait fallu répéter à des époques différentes de l'année pour voir l'évolution des données dans l'espace et dans le temps (cette opération trop coûteuse a dû être abandonnée à regret) ;

- d'autre part, l'étude de l'évolution spatio-temporelle de l'humidité dans le sol à l'aide d'un appareil mesurant la résistivité des terrains de surface (méthode électromagnétique). L'appareil n'ayant pu être acquis qu'au printemps 1990 grâce à des crédits obtenus du Réseau Européen des bassins-versants représentatifs et expérimentaux, l'expérience n'a pu être menée à temps. Cette étude sera effectuée en 1991 par le Laboratoire de la Montagne Alpine, l'intérêt de l'opération étant évident pour les participants au contrat et présentant l'originalité d'être la première à être menée sur l'ensemble d'un bassin-versant qui, à ce titre, mérite tout à fait son qualificatif d'expérimental.

Est-ce à dire qu'il est possible en ce site, de craindre plus des mouvements de versants, arrachements, glissements, etc... qu'une lave torrentielle nécessitant un apport de matériau dans le lit, certes, mais aussi un débit liquide suffisant pour l'entraîner?

\section{2 - Erosion torrentielle - instabilité des versants}

En l'absence de moyens de reconnaissance importants (sondages mécaniques, prospection géophysique lourde), notre étude analytique et synthétique a pu cependant aboutir à une cartographie géomorphologique détaillée et à la détermination des principaux critères d'instabilité des terrains et des milieux dans le secteur des Arcs. Cette étude devrait être complétée ultérieurement par une meilleure connaissance des épaisseurs des différentes couches reconnues en surface (géophysique et sondages) et par la mesure in situ et à long terme des processus morphodynamiques (ruissellement, solifluxion, glissement).

\section{A l'issue de l'étude, plusieurs constats s'imposent :}

(1) L'instabilité actuelle n'est pas catastrophique, mais plusieurs secteurs présentent des formes dynamiques ou d'autres indices hydrologiques et biogéographiques, qui révèlent des processus actifs (dont les vitesses d'évolution restent à préciser).

(2) Le bassin moyen de l'Eglise dans son ensemble (1900$1400 \mathrm{~m}$ ) et, dans le détail, les zones du Fevet, l'interfluve aval d'Arcs 1800 et le versant de l'Arpette-les Espagnols cumulent les indices et facteurs d'instabilité actuelle. Les versants les plus instables sont manifestement taillés dans les dépôts morainiques isérois (surtout s'ils sont plus riches en matrice gneissique altérée qu'en schistes) ou dans les schistes noirs argileux. En outre, le substrat schisteux apparaît altéré sur une grande profondeur, fissuré ou fracturé, et la tranche supérieure (10 à $40 \mathrm{~m}$ au moins) n'est pas en place dans ce bassin moyen.

(3) Le chenal du ruisseau de l'Eglise exhibe plusieurs segments de torrent «normalement actif » (charriage, incision, sapement des berges). Un seul segment, au niveau du Fevet $(1700-1480)$ se révèle en limite de stabilité (dissection linéaire, affouillement, sapement des berges affaissées). L'enfoncement du lit pourrait remettre en mouvement des glissements de terrain lents, surtout en rive droite (1650-1 $500 \mathrm{~m})$, d'autant plus que le fond du chenal pourrait ne pas être inscrit dans le substratum sain. En effet, lorsqu'ils affleurent, les schistes sont altérés, fissurés et ameublis sur une tranche très épaisse (plusieurs mètres à plusieurs dizaines de mètres). Le point sur les épaisseurs devrait faire l'objet d'une reconnaissance complémentaire sur ce site.

(4) De 1987 à 1990, aucune crise rhexistasique ne s'est produite, ni dans le chenal, ni sur les versants. Cependant, plusieurs formes dynamiques et mouvements de terrain, bien que modestes, ont été rendus actifs durant ou après les précipitations d'hiver (notamment les 10-16 et 26-28 février 1990) : coulée de débris sous l'Arpette $\left(750 \mathrm{~m}^{3}\right)$, arrachement-glissement à l'amont du Bérard $\left(300 \mathrm{~m}^{3}\right)$, quelques affaissements (dizaines à une centaine de $\mathrm{m}^{3}$ ) sur les deux rives du chenal au niveau du Fevet et de très nombreux éboulements ou arrachements de petite taille le long de la route départementale.

\section{(5) L'impact anthropique est sensible à trois niveaux :}

- les pistes de ski non reverdies du gradin subalpin et de la crête quartzitique, qui doivent modifier les circulations superficielles et les zones d'infiltration;

- les talus de la route départementale en limite de stabilité, les remblais et déblais des routes forestières;

- les surfaces bâties et recouvertes d'Arc 1800 constituent un impluvium conséquent pour les écoulements superficiels et souterrains qui convergent vers les nappes des matériaux morainiques et des schistes altérés déjà saturés, notamment sur les croupes instables des interfluves.

Dans les conditions actuelles d'un accroissement des interventions anthropiques, une accentuation des déséqui- 
libres localisés est à craindre, sans toutefois qu'une crise rhexistasique soit à redouter si ne survient pas un événement météorologique exceptionnel (intensité journalière de plus de $70 \mathrm{~mm}$; cf. ci-dessous).

\section{Les conditions de déclenchement d'une coulée de débris}

Le déclenchement d'une coulée de débris du type de la Ravoire dépend d'une convergence de facteurs d'instabilité, rarement réalisée (coulée dévastatrice 1 à 2 fois par siècle en moyenne Tarentaise ?). Ce déclenchement paraît nécessiter un ensemble de conditions nécessaires aggravant les facteurs naturels permanents.

Les Conditions nécessaires, facteurs naturels aggravants, sont :

(1) une séquence pluviométrique de quelques jours, dont le total dépasse largement le seuil d'intensité maximum observé en période estivale $(70 \mathrm{~mm} /$ jour, récurrence séculaire probable) et dont au moins deux subtotaux quotidiens atteignent ou dépassent le seuil d'intensité moyen observé (33-35 mm/jour). La séquence des 29 , 30 et 31 mars 1981 (total $98,7 \mathrm{~mm}$ ), s'ajoutant à la fonte nivale et celle (neigeuse) des 10-16 février 1990 (total $218 \mathrm{~mm}$ ) en constituent des exemples. La seconde n'a donné lieu qu'à des mouvements de terrain de faible ampleur cependant, car d'autres facteurs étaient absents de cette conjoncture ;

(2) un manteau neigeux épais, soit précoce (chute de 40$50 \mathrm{~cm} /$ jour en octobre-novembre), soit prolongé (marsavril) à moyenne altitude (100 à $180 \mathrm{~cm}$ d'épaisseur entre 1600 et $1800 \mathrm{~m}$ d'altitude au 29 mars 1988). En outre, ce manteau neigeux est instable s'il est tombé en abondance (en début d'hiver) ou s'il est profondément transformé (en fin d'hiver) sur un sol dégelé ;

(3) des températures moyennes plus élevées que les normales saisonnières à moyenne et haute altitude, par exemple, par type de temps doux du SSW avec vent chaud et foehn (cas des 29-31 mars 1981). Elles accentuent ou accélèrent la fusion de la neige, modifient la texture du manteau neigeux (à l'instar des précipitations plus tièdes que froides), ou peuvent conduire à son ressuyage brutal ;

(4) une recrudescence pluviométrique en fin de séquence de précipitations élevées (cas du 31 mars 1981). Des intensités de 5 à $10 \mathrm{~mm} / \mathrm{h}$ ont été couramment enregistrées aux Arcs lors des précipitations de $30 \mathrm{~mm} /$ jour. La récurrence d'une forte intensité pluviométrique de saison chaude $(60 \mathrm{~mm} / \mathrm{jour})$ serait de 3 fois par siècle et celle d'une très forte intensité $(70 \mathrm{~mm} / \mathrm{jour})$ d'une fois par siècle. Plus déterminante encore serait la durée (plusieurs heures) de la forte intensité horaire ( $>10 \mathrm{~mm} / \mathrm{h}$ ) pendant la journée de «forte précipitation " qui clôt la séquence pluviométrique " exceptionnelle " (rappelons à cet égard les $93 \mathrm{~mm}$ tombés en 90-180 mm au Grand-Bornand le 14.07.1987).

La combinaison des facteurs aggravants précédents agit comme une exceptionnelle interaction entre la séquence pluviométrique et la fonte nivale qui imbibe et sature les terrains, la recrudescence pluviométrique et l'accélération de la fusion nivale qui sature les nappes. S'y ajoute encore la circulation souterraine différée à la fois sur le bassinversant et provenant de celui d'Arc 2000, qui augmente rapidement la pression interstitielle d'une épaisse tranche de terrains décomprimés.

Les facteurs aggravants exercent un effet d'entraînement sur les facteurs naturels permanents, d'extension spatiale et à critères d'instabilité spécifiques :

(1) un substrat qui n'est ni sain ni en place, déjà imbibé, puis saturé, surtout s'il s'agit d'un matériau superficiel épais (10-40 m), meuble, sans cohésion ou pulvérulent et de texture sablo-argileuse. C'est le cas des dépôts morainiques isérois altérés (plus gneissiques que schisteux et sablo-argileux) et/ou des schistes noirs " argileux » altérés (cohésion nulle, angle de frottement interne moyen, indice de plasticité faible, argiles moyennement actives). C'est aussi le cas des remblais de route qui introduisent une surcharge sur le versant susceptible d'entraîner une rupture à leur aval (exemple probable à proximité de la cicatrice majeure du glissement de terrain à l'amont de la Ravoire) ;

(2) un arrachement-glissement ou un éboulement de ce type de matériau et son déplacement brutal vers le chenal du torrent. La masse saturée et meuble est prise en charge et incorporée à l'écoulement liquide, dont les propriété sont modifiées. L'incorporation de matériau est le facteur principal du déclenchement de la coulée de débris et de l'accroissement de son pouvoir érosif sur le lit et les berges du torrent. A cet égard, les matériaux glissés et instables des rives du torrent dans la zone du Fevet $(1700-1500 \mathrm{~m})$ et ceux issus d'un dépavage du lit constituent la charge solide incorporable, dans un scénario de grosse coulée de débris (ces volumes incorporables seront calculés en 1991 à partir d'un M.N.T. et des épaisseurs visibles ou sondées dans la Ravoire).

Un autre facteur, hydrogéologique, à la fois permanent et aggravant, est encore mal cerné, en particulier le rôle des "nappes perchées" et des écoulements hypodermiques dans les dépôts morainiques, ainsi que le rôle des circulations souterraines différées dans les massifs fissurés (surtout du gradin supérieur et du versant d'Arc 2000 vers le bassin moyen).

Toutefois, les observations de terrain détaillées, la cartographie thématique et intégrée et le repérage des indices, critères et facteurs de plusieurs types d'instabilité nous permettent de proposer une cartographie complémentaire et synthétique (par rapport aux précédentes) dans le domaine des risques liés à une instabilité potentielle. Notre programme pour l'année 1991 prévoit cette action qui sera guidée par la prise en compte des notions de vulnérabilité, de patrimoine et, surtout, de deux scénarios:

- le déclenchement d'une coulée de débris (calcul des volumes mobilisables);

- la mobilisation de versants déformés et glissés (calcul de stabilité et des volumes incorporables). 